\title{
Curing Monitoring of Graphite/Epoxy Laminated Composite Material by Using Embedded Corrugated Long-Period Fiber Grating Sensor
}

\author{
Chein-Jung Wu, Liren Tsai, Tsung-Chieh Cheng, Bo-Lan Fang, and Chia-Chin Chiang* \\ Department of Mechanical Engineering, National Kaohsiung University of Applied Sciences, \\ 415 Chien Kung Road, Kaohsiung 807, Taiwan (R.O.C.)
}

(Received January 9, 2015; accepted January 8, 2016)

\begin{abstract}
Keywords: composite materials, residual stress, corrugated long-period fiber grating, curing monitoring
\end{abstract}
The curing monitoring of polymeric composite materials is necessary to improve the mechanical performance, especially for the characterization of residual stress after manufacture. We aimed to explore the use of embedded corrugated long-period fiber gratings (CLPFG) to characterize the curing process in a graphite/epoxy composite material. The curing development and residual stress measurement were assessed through changes in the shape of the optical spectra, intensity attenuation, and shifts in wavelengths in the optical fiber sensors. By monitoring the spectra of the CLPFG during the curing process, the maximum axial residual stress induced by the curing process was $35 \mathrm{MPa}$ in the composite material.

\section{Introduction}

Carbon-fiber-reinforced plastics (CFRP) have good specific strength, and are used in various fields such as aircraft and aerospace applications. ${ }^{(1)}$ The curing monitoring of the polymeric composite materials has attracted wide interest recently. ${ }^{(2)}$ Monitoring the curing process is necessary to improve the mechanical performance of graphite/epoxy composites. The long-period fiber grating (LPFG) is one such candidate finding increasing applications as a sensor in aerospace, structural, medical and chemical applications for temperature, strain, and general structural health monitoring. ${ }^{(3,4)}$

LPFG sensors have a lot of advantages such as electrically passive operation, light weight, immunity to electromagnetic interference, corrosion resistance, and high temperature resistance. Therefore, optical fiber sensors have been extensively applied to various fields. LPFG sensors are small and compatible with common polymeric materials. They can be easily embedded close to the internal sensing site in a composite structure without introducing significant defects. Therefore, the LPFG is suitable for application as an embedded sensor for CFRP materials.

In 1998, Du et al. ${ }^{(5)}$ embedded the LPFG into glass fiber/polyester resin composites for threepoint bending measurement. The three-point bending measurements after embedding reveal that when the bending radius ranges from 0 to $0.001 / \mathrm{mm}$, the optical energy of the LPFG penetrating the spectrum declines linearly, indicating that the bending radius is inversely related to the optical energy within the spectrum.

"Corresponding author: e-mail: ccchiang@kuas.edu.tw 
In 2001, Dunkers et al.(6) proposed using the LPFG to monitor resin flow during liquid composite molding (LCM) and the effect of curing fiber-reinforced glass on LPFG. They determined that the resonance spectrum disappeared when the refractive index was higher than that of the LPFG covered by resin-fiber cladding and that the stress of the fiber-reinforced glass on the LPFG contributed to the gradual disappearance of the resonance spectrum.

In 2005, Zhu et al. (7) used the LPFG to monitor the effect of varying the refractive indices and viscosities of resin during LCM on the resonance spectrum. They observed that the resonance wavelength shifted toward the left as the resin viscosity and refractive index increased and that the resonance spectrum completely disappeared when the refractive index of the resin exceeded that of the fiber cladding. In 2006, Tan et al. ${ }^{(8)}$ reported on the LPFG embedded into photosensitive fibers by using an excimer laser with an amplitude mask and embedded the LPFG into composite carbon fibers for curing. After curing, the specimens were examined using a three-point bending technique to explore the relationship between the bending curvature and resonance amplitude depth of the LPFG. When the curvature was within the range of $0-2 \mathrm{~m}^{-1}$, the resonance amplitude depth increased linearly and the position of the resonance wavelength remained unchanged, at a sensitivity of $5.065 \mathrm{dBm}^{-1}$. In 2007, Buggy et al. ${ }^{(9)}$ monitored variations in refractive index during resin curing by employing three fiber optic sensors, namely the LPFG, tilted Bragg gratings, and the Fresnel refractometer. Their results revealed that the refractive indices measured using the LPFG and the Fresnel refractometer were highly consistent. In 2007, Buggy et al. ${ }^{(10)}$ simultaneously reported on the two long-period grating embedded into optical fibers by using the phase mask technique to form a Mach-Zehnder interferometer, which was subsequently used to monitor the curing process of epoxy. After Fourier transformation, the experimental results indicated that the Fourier function of the frequency amplitude was inversely proportional to variations in the resin refractive index.

In 2001, Lin et al. ${ }^{(11)}$ were the first to report on the use of an etching technique for the fabrication of a corrugated LPFG (CLPFG). The diameter of the CLPFG is smaller than that of the normal LPFG. Therefore, the CLPFG is more suitable for embedded sensor application. On the basis of the aforementioned studies on the LPFG used to monitor composites and curing, much of the research focuses on monitoring the refractive index variation of polymer resin or on embedding the LPFG into a composite material to measure bending curvature. However, little effort has been invested in embedding the LPFG into composite materials to monitor the curing process in real time. To fill this gap, the current study involved monitoring the curing process by applying a CLPFG produced by hydrofluoric acid etching. The CLPFG structure is generated on the surface of the fiber cladding, causing the resonance attenuation to vary according to the changing temperature, strain, and refractive index. The curing development and residual stress measurement were assessed through changes in the optical spectra of the optical fiber sensors. The curing residual stress is presented and analyzed systematically in this paper.

\section{Theory}

In this study, a periodic structure was produced on the surface of fiber cladding through a wet etching process, and the number of grating periods ranged between 100 and $1000 \mu \mathrm{m}$. When light propagates within the fiber grating, periodic gratings cause the light to be coupled from the core mode to the cladding mode and produce a resonant dip in the transmission spectrum.

The CLPFG resonance attenuation wavelength can be calculated using the following formula: ${ }^{(11,12)}$ 


$$
\lambda=\left(n_{\mathrm{eff}}^{\mathrm{co}}-n_{\mathrm{eff}}^{\mathrm{cl}}\right) \Lambda
$$

where $\lambda$ represents the resonance wavelength, $\Lambda$ is the grating period, $n_{\text {eff }}^{\mathrm{co}}$ is the effective refractive index of the core, and $n_{\text {eff }}^{\text {cl }}$ is the effective refractive index of the cladding. The transmission loss of LPFG is defined as ${ }^{(11,12)}$

$$
T=\cos ^{2}\left(\kappa_{\text {core-cladding }}^{\text {ac }} L\right)
$$

where $\kappa_{\text {core-cladding }}^{\text {ac }}$ represents the ac coupling coefficient between the core mode of the cladding mode. According to Eq. (1), the LPFG resonance wavelength is inversely proportional to the grating periods, whereas transmission loss has the distribution of a cosine-squared function.

\section{Experimental Method}

\subsection{Processing and fabrication}

The materials used in this study were thermoset composite materials, reinforced carbon fibers, and an epoxy matrix. The curing and molding conditions are shown in Fig. 1. The temperature was increased to $150{ }^{\circ} \mathrm{C}$ at a rate of $3{ }^{\circ} \mathrm{C} / \mathrm{min}$, stopped for $30 \mathrm{~min}$, and then allowed to naturally cool. The air pressure was $6 \mathrm{~kg} / \mathrm{cm}^{2}$, and the vacuum pressure was $76 \mathrm{~cm} / \mathrm{Hg}$.

The CLPFG used in this study was processed using buffered oxide etch (BOE) to change the diameter of the fiber by etching. The steps of the fiber etching procedure, illustrated in Fig. 2(a), are discussed as follows: First, approximately $3 \mathrm{~cm}$ of the protective layer is removed at the center of a single-mode fiber section, and the fiber is adhered to the etching board, which is then placed into a plastic box into which BOE is poured for etching. Subsequently, a second etching is performed using wet etching. As illustrated in Fig. 2(b), the wet etching consists of several steps discussed as follows: First, copper plating is sputtered on a wafer, an initial layer of photoresist is applied to generate the first layer of a period structure $(650 \mu \mathrm{m})$ through exposure and development, and the previously etched fiber is pasted onto the wafer. Then, the second coating layer of photoresist SU-8 3050 is spun again to cover the optical fiber and develop a second etch.

At this point, the diameter of the unprotected portion of the fiber is altered by the second etch. When the etched optical fiber $(45 \mu \mathrm{m})$ is completed, the fiber is delaminated using ferric chloride and soaked in sulfuric acid before being removed for use.

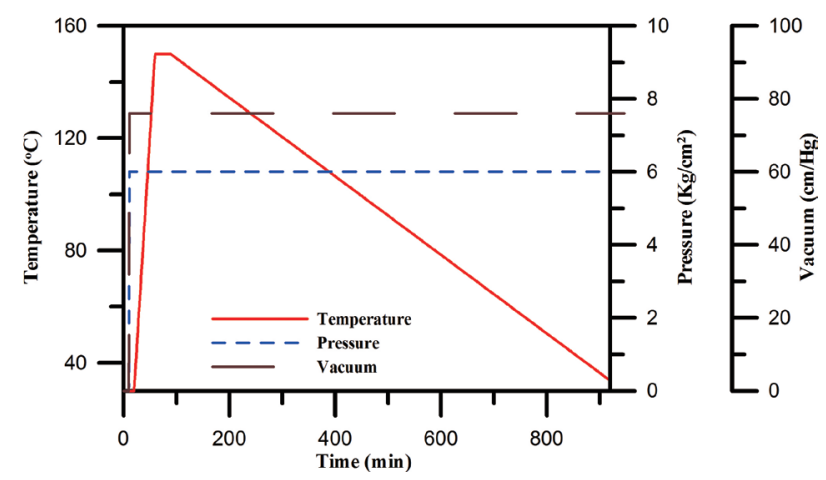

Fig. 1. (Color online) Curing conditions. 


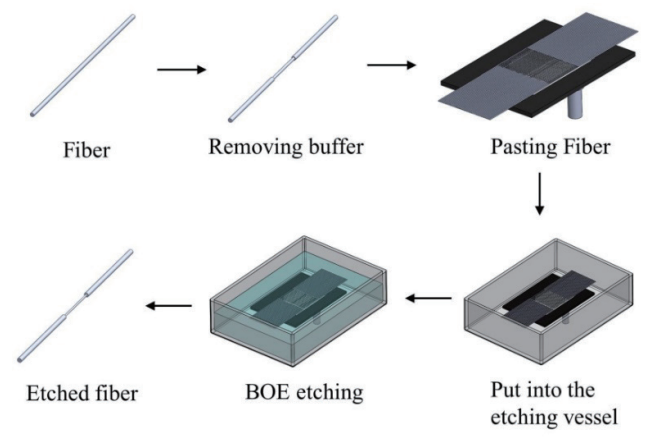

(a)

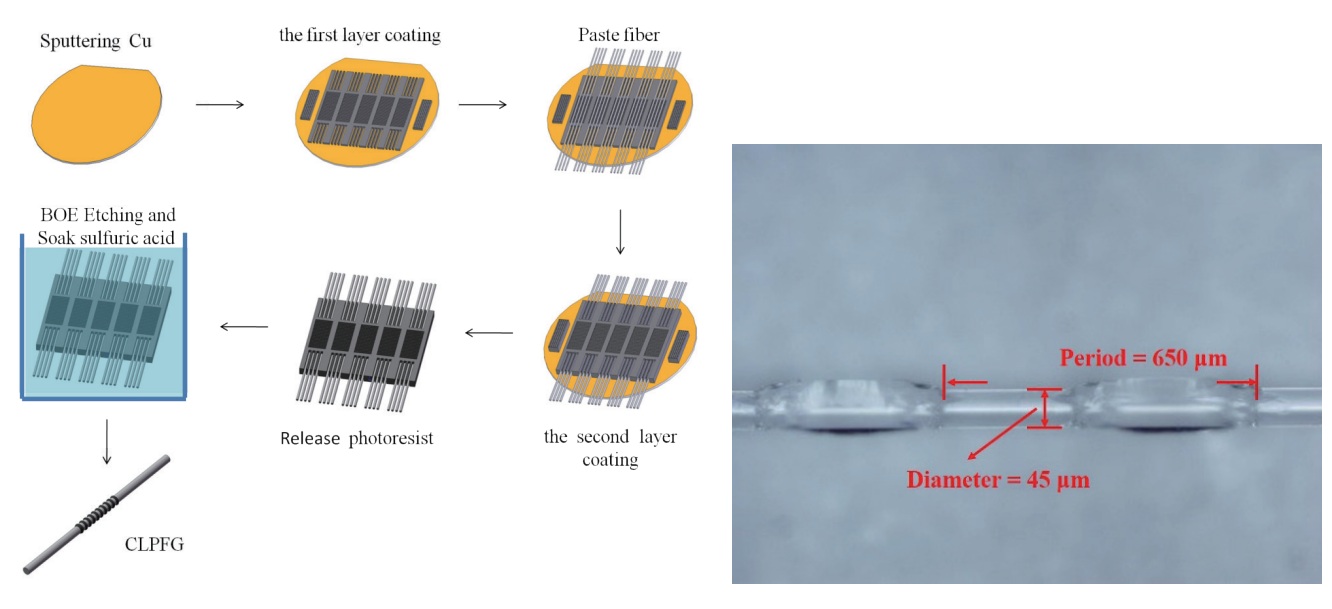

(b)

Fig. 2. (Color online) (a) Fiber etching procedure. (b) Fabrication process of CLPFG.

\subsection{Experimental setup for composite curing monitoring}

The CLPFG was embedded between layers 8 and 9 of the prepreg material in the following stacking order: [ $90^{\circ} / 0^{\circ} / 90^{\circ} / 0^{\circ} / 90^{\circ} / 0^{\circ} / 90^{\circ} / 0^{\circ} /$ \{ Sensor $\} / 0^{\circ} / 90^{\circ} / 0^{\circ} / 90^{\circ} / 0^{\circ} / 90^{\circ} / 0^{\circ} / 90^{\circ}$ ].

The symmetry of the 16-layer structure was maintained by placing the first sheet of Teflon fabric, followed by the prepreg material, a second sheet of Teflon fabric, high-temperature diaphragm, and O-rings into the mold, which was placed into the thermal chamber by using an air compressor and vacuum pump for thermoforming. Figures 3 and 4 show the experimental processes of CLPFG embedding and curing monitoring.

\section{Results and Discussion}

\subsection{Force calibration of the CLPFG sensor}

Figure 5 illustrates the spectra of the CLPFG sensor under various tensile loading conditions ( 0 $0.4214 \mathrm{~N}$ ). The development of transmission dips can be observed from the spectra of the CLPFG with various strains. Based on the figure, when the strain loading of the CLPFG increases, the 

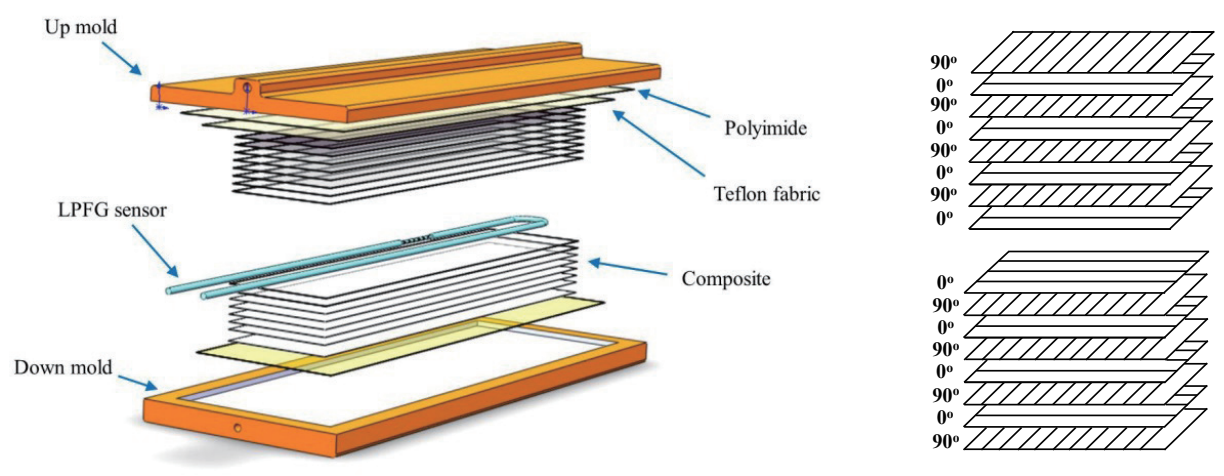

Fig. 3. (Color online) Schematic diagram of CLPFG embedding.

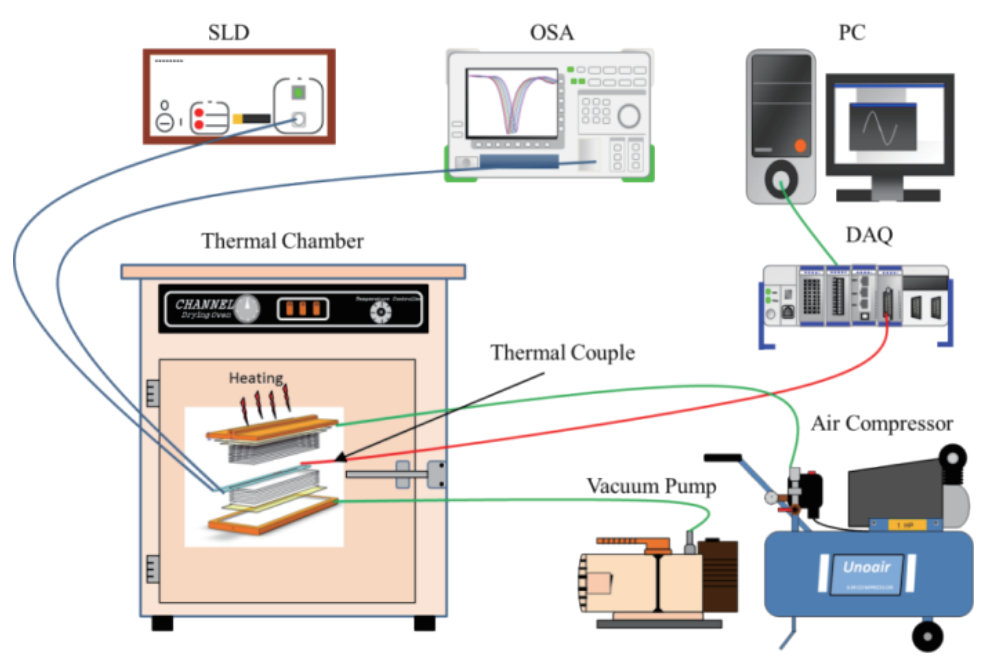

Fig. 4. (Color online) Setup of the composite curing monitoring with embedded CLPFG sensor.

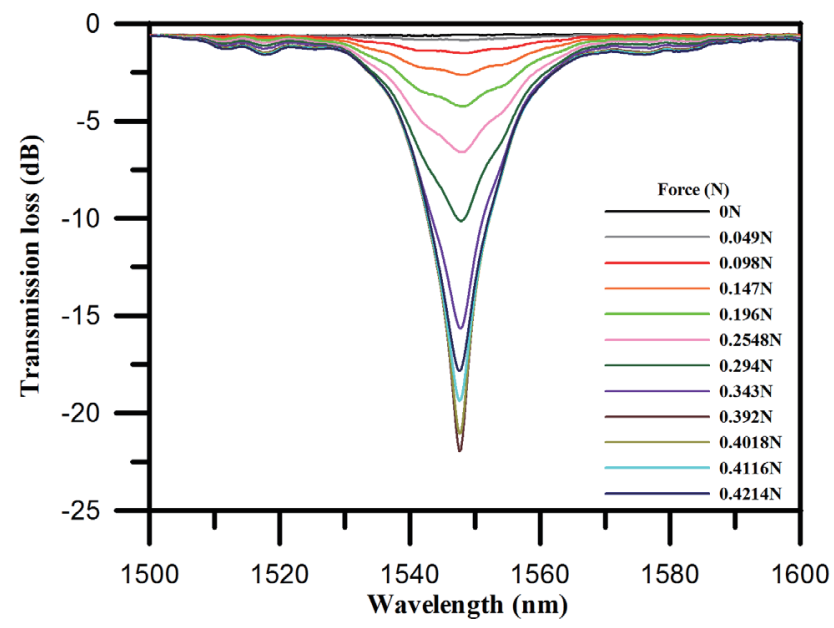

Fig. 5. (Color online) Force calibration spectra of CLPFG sensor. 
transmission dip will deform and grow with various strains. The dips of the CLPFG grow linearly up to the maximum transmission loss. The maximum transmission dip of the CLPFG is $-21.94 \mathrm{~dB}$ at $1547.6 \mathrm{~nm}$ under an applied tensile loading of $0.4214 \mathrm{~N}$.

\subsection{Monitoring curing of CFRP composite materials by CLPFG sensor}

The curing process includes heating, isothermal, and cooling stages. At the heating stage, the temperature rises from 25 to $150{ }^{\circ} \mathrm{C}$. Figure 6 shows the spectra of the CLPFG sensor during the heating stage of the curing process. At $30^{\circ} \mathrm{C}$, a resonance attenuation peak was generated as a result of the pressurizing effect of the air compressor and vacuum pump, and the transmission loss was $-2.33 \mathrm{~dB}$. Regarding the force calibration results, the tensile force was less than $0.147 \mathrm{~N}$. Throughout the heating process, the spectrum of light energy underwent little variation; however the variation was more prominent at only long wavelength bands. The resonance attenuation peak showed a downward trend before $50{ }^{\circ} \mathrm{C}$, but rebounded after $50{ }^{\circ} \mathrm{C}$, subsequently shifting gradually toward long wavelengths.

Figure 7 shows the variations of temperature-transmission loss during the heating stage of the curing process. The transmission loss revealed a diminishing trend before $50{ }^{\circ} \mathrm{C}$, presumably caused by the pressurizing effect of the air compressor and vacuum pump. After $50{ }^{\circ} \mathrm{C}$, the diminishing trend begins to rebound, presumably because of the decreased pressure on the CLPFG by the air compressor, vacuum pump, and resin, when the resin transforms from melted liquid into a gel state. When the temperature reaches $80^{\circ} \mathrm{C}$, the increasing trend slows until $115^{\circ} \mathrm{C}$. This trend is presumably linked to the curing cross-linking reaction.

After the heating and isothermal stages, the composite is cooled from $150{ }^{\circ} \mathrm{C}$ to room temperature. Figure 8 shows the spectra of the embedded CLPFG sensor during the cooling process. When the temperature decreased from 150 to $130{ }^{\circ} \mathrm{C}$, the resonance attenuation peak exhibited a shoaling trend, which changed into a deepening trend from 130 to $30{ }^{\circ} \mathrm{C}$. When the temperature reached $30{ }^{\circ} \mathrm{C}$, the transmission loss reached its minimum at $-3.95 \mathrm{~dB}$. Throughout

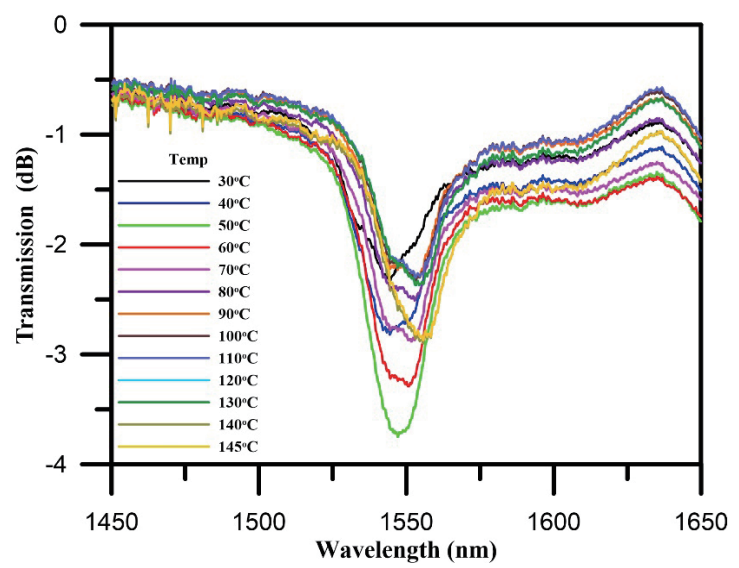

Fig. 6. (Color online) Spectra of the CLPFG sensor during heating stage of curing process.

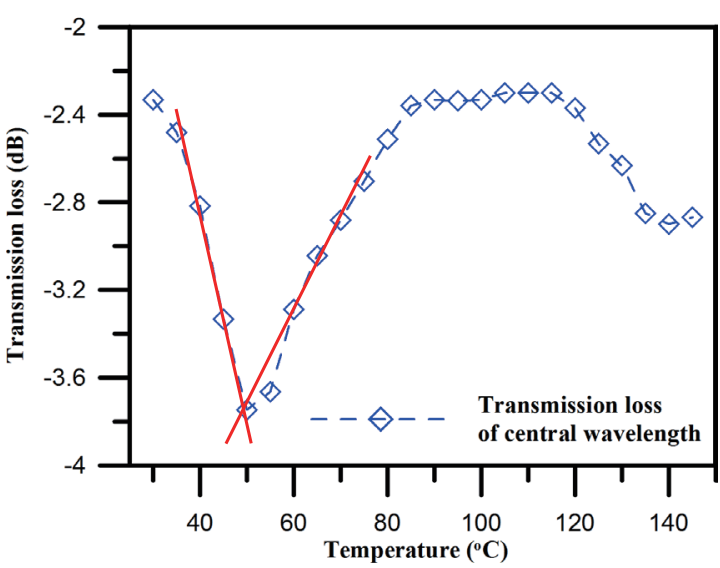

Fig. 7. (Color online) Temperature-transmission loss relationship during heating stage of curing process. 


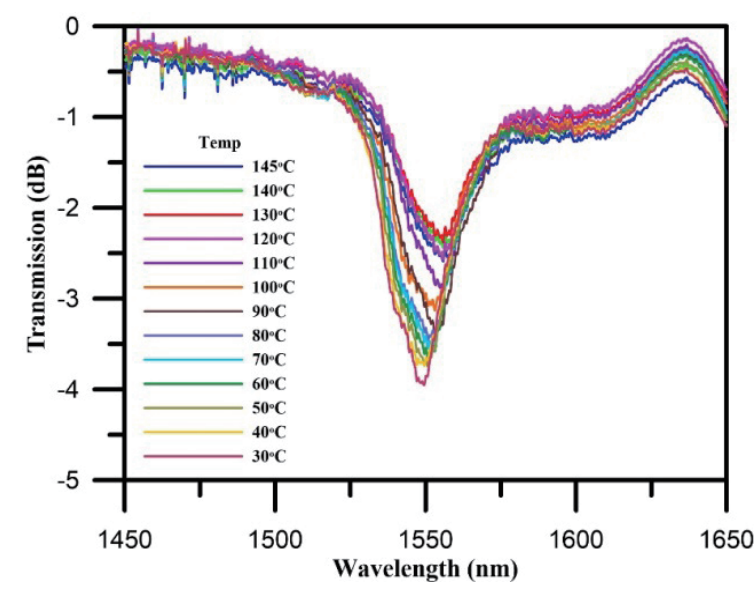

Fig. 8. (Color online) Spectra of embedded CLPFG sensor during the cooling process.

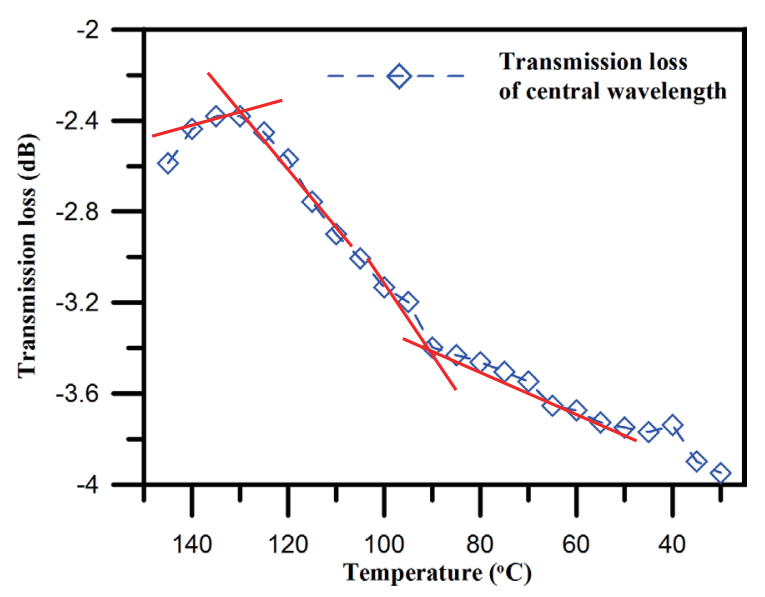

Fig. 9. (Color online) Change in transmission loss of CLPFG sensor with temperature during the cooling process.

the cooling process, the light energy in the spectrum was fairly stable, except for a few minor variations, and the spectrum exhibited a slow trend of shifting toward short wavelengths.

An observation of the variations in transmission loss (Fig. 9) reveals that the CLPFG sensor's transmission loss begins to decrease after $130{ }^{\circ} \mathrm{C}$; thus, the residual stress can be inferred to occur at $130{ }^{\circ} \mathrm{C}$. After $80{ }^{\circ} \mathrm{C}$, the decreasing slope of transmission loss slows, and when the CLPFG sensor cools to room temperature, its transmission loss is $-3.95 \mathrm{~dB}$. Regarding the results of force calibration, the internal residual force may be approximately $0.237 \mathrm{~N}$. By executing a crosssectional area conversion of the etched fiber, the residual stress of the CLPFG sensor was about 35 $\mathrm{MPa}$.

\section{Conclusions}

In this study, we successfully monitored the curing process of a composite material by using an embedded CLPFG sensor. The experimental results reveal that during the heating process of the composite material curing, optical spectrum variations of the CLPFG are observable at approximately $50{ }^{\circ} \mathrm{C}$. This phenomenon may be attributed to the effect of pressure loading on the CLPFG when the composite epoxy matrix undergoes a gelation reaction at the gelation temperature. When the temperature reaches $80{ }^{\circ} \mathrm{C}$, the increasing trend slows until $115^{\circ} \mathrm{C}$. This trend is linked to the curing cross-linking reaction. In the cooling process, the transmission loss of the embedded CLPFG sensor begins to decrease after $130{ }^{\circ} \mathrm{C}$. Therefore, the residual stress can be inferred to occur at $130{ }^{\circ} \mathrm{C}$. Finally, based on the force calibration, the average residual stress was measured with the CLPFG, and the axial residual stress was about $35 \mathrm{MPa}$.

\section{Acknowledgments}

This work was supported by the Ministry of Science and Technology, Taiwan (grant number MOST 103-2221-E-151-009-MY3). 


\section{References}

1 Y. Okabe, S. Yashiro, R. Tsuji, T. Mizutani, and N. Takeda: Composites Part A 33 (2002) 991.

2 V. M. Murukeshan, P. Y. Chan, L. S. Ong, and L. K. Seah: Sens. Actuators, A 79 (2000) 153.

3 G. Rego: Sci. World J. 2013 (2013) 14.

4 A. Martinez-Rios, D. Monzon-Hernandez, and I. Torres-Gomez: Opt. Commun. 283 (2010) 958.

5 W. Du, H. Y. Tam, M. S. Y. Liu, and X. Tao: Proc. SPIE—The International Society for Optical Engineering, Smart Structures and Materials 1998: Sensory Phenomena and Measurement Instrumentation for Smart Structures and Materials (San Diego, CA, United States, 1998) Vol. 3330, pp. 284-292.

6 J. P. Dunkers, J. L. Lenhart, S. R. Kueh, J. H. Van Zanten, S. G. Advani, and R. S. Parnas: Opt. Laser Eng. 35 (2001) 91.

7 Y. Zhu, C. Wang, J. Wang, W. He, and G. Gao: J. Mater. Sci. Technol. 21 (2005) 234.

8 K. M. Tan, C. C. Chan, S. C. Tjin, and X. Y. Dong: Sens. Actuators, A 125 (2006) 267.

9 S. J. Buggy, E. Chehura, S. W. James, and R. P. Tatam: J. Opt. A: Pure Appl. Opt. 9 (2007) S60.

10 S. J. Buggy, R. P. Murphy, S. W. James, and R. P. Tatam: Proc. SPIE-The International Society for Optical Engineering, Third European Workshop on Optical Fibre Sensors (Napoli, Italy, 2007) Vol. 6619, p. 24.

11 C. Y. Lin, L. A. Wang, and G. W. Chern: J. Lightwave Technol. 19 (2001) 1159.

12 T. Erdogan: J. Lightwave Technol. 15 (1997) 1277. 